\title{
The prevalence and prognosis of hyponatraemia in non-Hodgkin lymphoma-associated hemophagocytic lymphohistiocytosis
}

\section{Guangli Yin}

The First Affiliated Hospital of Nanjing Medical University, Jiangsu Province Hospital, China Changfeng Man

The First Affiliated Hospital of Nanjing Medical University, Jiangsu Province Hospital, China Jiayu Huang

The First Affiliated Hospital of Nanjing Medical University, Jiangsu Province Hospital, China

\section{Wanying Cheng}

The First Affiliated Hospital of Nanjing Medical University, Jiangsu Province Hospital, China

\section{Xin Gao}

The First Affiliated Hospital of Nanjing Medical University, Jiangsu Province Hospital, China

\section{Jujuan Wang}

The First Affiliated Hospital of Nanjing Medical University, Jiangsu Province Hospital, China

\section{Tian Tian}

The First Affiliated Hospital of Nanjing Medical University, Jiangsu Province Hospital, China

\section{Limin Duan}

The First Affiliated Hospital of Nanjing Medical University, Jiangsu Province Hospital, China

Ji Xu

The First Affiliated Hospital of Nanjing Medical University, Jiangsu Province Hospital, China Hongxia Qiu ( qhx9805@126.com )

The First Affiliated Hospital of Nanjing Medical University, Jiangsu Province Hospital, China

\section{Research Article}

Keywords: Hemophagocytic lymphohistiocytosis, Non-Hodgkin lymphoma, Hyponatraemia, Prognosis, Survival

Posted Date: April 30th, 2021

DOI: https://doi.org/10.21203/rs.3.rs-386327/v2

License: (c) (1) This work is licensed under a Creative Commons Attribution 4.0 International License. 
Version of Record: A version of this preprint was published at Leukemia \& Lymphoma on October 18th, 2021. See the published version at https://doi.org/10.1080/10428194.2021.1992623. 


\section{The prevalence and prognosis of hyponatraemia in non-Hodgkin lymphoma-associated hemophagocytic lymphohistiocytosis}

Guangli Yin ${ }^{1 \#}$, Changfeng Man ${ }^{1 \#}$, Jiayu Huang ${ }^{1}$, Wanying Cheng ${ }^{1}$, Xin Gao ${ }^{1}$,

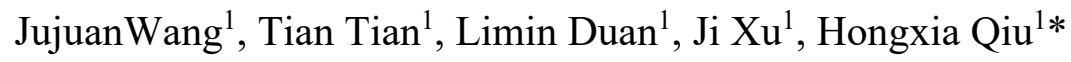

${ }^{1}$ Department of Geriatric Hematology, The First Affiliated Hospital of Nanjing Medical University, Jiangsu Province Hospital,300 Guangzhou Road, Nanjing 210029, China.

${ }^{\#}$ Guangli Yin and Changfeng Man contributed equally to this work.

\section{*Correspondence:}

Hongxia Qiu, E-mail: qhx9805@126.com, Department of Geriatric Hematology, The First Affiliated Hospital of Nanjing Medical University, Jiangsu Province Hospital, 300 Guangzhou Road, Nanjing 210029, China 
2 Background: Non-Hodgkin lymphoma associated hemophagocytic

3 lymphohistiocytosis (NHL-HLH) in adult secondary HLH (sHLH) is a common and

4 universally highly lethal critical disorder. Hyponatraemia is the most common

5 electrolyte disorder in the critical illness setting and acts as a negative prognostic factor.

6 The aim of our study was to evaluate the prognostic role of hyponatraemia among

7 patients with NHL-HLH.

8 Methods: This retrospective study enrolled 153 newly diagnosed adult NHL-HLH patients, which were divided into 2 groups based on serum sodium concentration on admission, a hyponatraemia and a normonatraemia group. Univariate and multivariate Cox regression analysis were used to identify the prognostic factors associated with worse survival. The overall survival time of all the patients were compared using log rank tests. Restricted cubic splines were conducted to address the association between serum sodium concentration and the risk of mortality.

Results: The results showed that $81(52.9 \%)$ patients had hyponatraemia. After a median follow-up of 47 (range 14-180) days, there were 72 (88.9\%) cumulative deaths in hyponatraemia group while $50(69.4 \%)$ in normonatremia group. Univariate Cox regression analysis showed that hyponatraemia group demonstrated unfavourable overall survival $(P=0.002)$. After adjustment for confounders, multivariate analysis revealed that hyponatraemia was an independent prognostic factor for OS (Hazard ratio [HR]:1.51, 95\% confidence interval [CI]: 1.03-2.20; $P=0.033)$. Restricted cubic spline confirmed a linear and positive association between serum sodium and the risk of mortality.

Conclusions: Hyponatraemia is relatively frequent in NHL-HLH. As a readily available biomarker in clinical routine, it was a promising prognostic predictor for NHL-HLH.

Keywords: Hemophagocytic lymphohistiocytosis, Non-Hodgkin lymphoma, Hyponatraemia, Prognosis, Survival 


\section{Background}

Hemophagocytic lymphohistiocytosis (HLH), categorized as genetic or acquired, is considered to be a life-threatening syndrome characterized by aberrant activation and proliferation of polyclonal $\mathrm{T}$ lymphocytes and mononuclear macrophages, which leads to an uncontrolled hyperinflammatory response. Adult secondary HLH is commonly triggered by infections, malignancies, autoimmune disorders or unknown aetiologies[1]. Of these, malignancy is the most common trigger identified in adult HLH, especially non-Hodgkin lymphoma-associated sHLH (NHL-HLH), which has a high mortality rate and is considered a major challenge to clinicians due to sHLH and lymphoma share main aspects[2]. NHL-HLH has a progressive course, with mortality rates ranging from $20 \%$ to $60 \%[3,4]$. Early identification of risk factors among NHL-HLH patients is essential for appropriate treatment strategies that rapidly control hyperinflammation and hypercytokine responses.

Hyponatraemia, defined as a serum sodium level $<135 \mathrm{mmol} / \mathrm{L}$, is the most common electrolyte disturbance in critically ill patients and cancer patients. Studies have also shown that hyponatraemia is associated with inferior survival of critical illnesses and various types of cancers, including heart failure[5], shock [6], SLE [7], breast cancer (BC), colorectal cancer (CRC), lung cancer and lymphoma [8]. However, no previous literature has investigated the prognostic value of hyponatraemia in NHLHLH patients on admission.

The purpose of our study was to explore the incidence of hyponatraemia and investigate the relationship between hyponatraemia on admission and overall survival 
among adult onset NHL-HLH patients.

\section{Methods}

\section{Study patients}

A total of 153 consecutive patients newly diagnosed between January 1, 2015, and December 28, 2019, at our hospital were included. The inclusion criteria were as follows: 1) patients with an age more than 18 years old; 2) patients for whom the diagnosis of NHL met WHO pathological criteria for biopsy samples [9] or patients for whom the diagnosis of NHL was based on MICM (morphology, flow cytometric immunophenotype, IgH or TCR rearrangement and immunohistochemistry of bone marrow biopsy) criteria [10]; and 3) patients who fulfilled at least 5 of the 8 criteria proposed by the Histiocyte Society in 2004 before treatment [11]. The exclusion criteria were as follows: 1) patients with previous illnesses (i.e. heart, liver or renal diseases); 2) patients with history of medication (i.e. antihypertensives or antidepressants); 3) progressive multiple organ dysfunction (MODS) before treatment; 4) patients with a serum sodium over $145 \mathrm{mmol} / \mathrm{L}$; and 5) patients who acquired HLH during chemotherapy or refused any treatment (Fig. 1).

\section{Parameters associated with NHL-HLH}

Clinical parameters assessed at the first admission included fever, complete blood cell counts, blood biochemical tests including triglycerides (TG), lactate dehydrogenase (LDH), albumin (ALB), fibrinogen (FIB), ferritin, and serum soluble interleukin-2 receptor (sIL-2R, sCD25). Hscore criteria were further applied to support the diagnosis of HLH based on HLH-2004 criteria [12]. We did not evaluate natural killer (NK) cell cytotoxic activity. EBV was evaluated by both serology and EBV DNA real-time 
quantitative polymerase chain reaction (RQ-PCR) analysis. Bone marrow aspiration and biopsy samples were reviewed at the first diagnosis. The type of initial therapy was also reviewed. For this analysis, patients were divided into two groups: hyponatraemia was defined as below $135 \mathrm{mmol} / \mathrm{L}$; normonatraemia between 135 and $145 \mathrm{mmol} / \mathrm{L}$. Hypernatraemia was excluded from the analysis because there were only two patients (147.5 mmol/L and $156 \mathrm{mmol} / \mathrm{L})$ in our collected clinical data.

\section{Statistical analysis}

Data analysis was performed using SPSS version 23.0 (Chicago, IL, USA) and MedCalc (version 15.6.1, Ostend, Belgium). To determine the equality of variances, Levine's test was used. Continuous variables are presented as the means \pm standard deviations or the medians (with interquartile ranges, IQR). We used Student's t-test for normally distributed variables and the Mann-Whitney $U$ test for non-normally distributed variables. Categorical data are presented as frequencies and percentages using Pearson's $\chi^{2}$ test. Overall survival (OS) was the primary outcome and was defined as the time from the first day of diagnosis to the date of death due to any cause or last follow-up. The association between hyponatraemia and overall survival was examined using Kaplan-Meier estimators and the log-rank test. To identify predictors of death, a Cox proportional hazards regression analysis was performed. Continuous variables (absolute neutrophil count, hemoglobin, platelet, fibrinogen, TG, triglyceride) optimal cut-off values of patients' clinical parameters were dichotomized applying usual clinical thresholds according to HLH-2004 protocol of the International Histiocyte Society in Univariate and multivariate Cox regression analyses. Optimal cut- 
off values of ALB and ferritin were dichotomized according to the serum albumin level and previous literature[13, 14]. Receiver operating characteristic (ROC) curve analysis was used to evaluate the ability of sCD25 to discriminate between survivors and nonsurvivors and to find the ideal cut-off value of sCD25. Variables $(P<0.05$ in univariate analysis) were entered into a Cox proportional hazards regression model with a stepwise variable elimination procedure in the multivariate model. Restricted cubic splines with three knots placed at the $10^{\text {th }}, 50^{\text {th }}$ and $90^{\text {th }}$ percentiles were generated to examine the nonlinear relationships of serum sodium with the risk of mortality after adjusting for confounding factors, and the tests for nonlinearity were calculated by Wald $\chi^{2}$ tests[15]. Moreover, we performed subgroup analyses to assess the interaction between hyponatraemia and clinically related and valuable variables by means of Cox proportional hazards analysis. A two-sided $P<0.05$ was used to define statistical significance for all comparison.

\section{Results}

\section{Serum sodium levels in NHL-HLH patients and correlations with patient clinical}

\section{parameters}

One hundred and fifty-three patients with detailed serum sodium profiles at diagnosis of NHL-HLH were recruited in our study, with a median age of 54 years (range, 41.564.5 years). The majority of sHLH patients were male (71.9\%). Hyperferritinaemia, elevated sCD25, fever, splenomegaly and lymphadenopathy were present in more than $95 \%$ of patients, whereas hepatomegaly and bone marrow hemophagocytosis were present in $36.6 \%$ and $86.3 \%$ of patients, respectively (Supplementary file1). All 
recruited patients were categorized as having B cell lymphoma-triggered HLH (BCLHLH) $(n=62,40.5 \%)$, of which the most frequent histopathological subtype was B cell lymphoma, unclassified, followed by diffuse large B cell lymphoma (DLBCL); T cell lymphoma-triggered HLH (TCL-HLH) was observed in 91 (59.5\%) patients, consisting of aggressive NK/T cell lymphoma and T cell lymphoma, unclassified.

The association between serum sodium $\left(\mathrm{S}-\mathrm{Na}^{+}\right)$concentration on admission and baseline clinical parameters of 153 NHL-HLH patients is summarized in Table 1. Four parameters were significantly elevated in patients with hyponatraemia compared with normonatraemia: ferritin levels $(P<0.001)$, the proportion of patients with EBV infection and the proportion of patients with TCL- HLH $(P=0.034$ and $P=0.01$, respectively). Only serum chlorine $\left(\mathrm{S}^{-} \mathrm{Cl}^{-}\right)$was significantly decreased in patients with distribution of NHL-HLH therapies between the two groups. chemotherapy, including 70 patients treated with a $\mathrm{CEOP} \pm \mathrm{R}$-based regimen doxorubicin, etoposide, and methylprednisolone), 4 treated with the $\mathrm{P}-\mathrm{GemOx} \pm \mathrm{R}$ regimen (pegaspargase, gemcitabine, oxaliplatin and/or ruxolitinib), 2 treated with anti- 
140 (rituximab, cyclophosphamide, doxorubicin, vincristine and dexamethasone alternating

141 with rituximab, high-dose methotrexate and cytarabine). A median of 1 cycle (range 1-

1426 cycles) was given. The other 43 patients received only the HLH-94 protocol or steroid

143 and/or etoposide therapies. Patients did not receive lymphoma-specific therapy is that

144 early death while receiving HLH directed therapy, not suitable for intensive treatment

145 due to MODS.

146 Hyponatraemia and overall survival

147 The median survival was 47 (16-180) days in all evaluable patients. A total of 122

148 (79.7\%) subjects died until the end of follow-up. The Kaplan-Meier method was

149 employed to estimate the prognostic significance of $\mathrm{S}-\mathrm{Na}^{+}$levels in NHL-HLH (Fig.

150 2A). Compared to normonatraemia, patients with hyponatraemia on admission showed

151 evidently worse overall survival (median: 92 days vs 30 days, $P=0.0018$ ). In the Cox

152 multivariate analysis, hyponatraemia (HR:1.51, 95\% CI: 1.03-2.20; $P=0.033$ )

153 remained independently associated with poor survival (Table 2). Restricted cubic spline

154 regression models revealed a significant linear relationship between serum sodium and

155 risk of mortality after adjusting for potential confounding factors (Fig. 2B). The effect

156 was flat when serum sodium was more than $135 \mathrm{mmol} / \mathrm{L}$ concentrations and was sharply

157 increased when less than $135 \mathrm{mmol} / \mathrm{L}$.

158 Subgroup evaluation of hyponatraemia in NHL-HLH

159 In the subgroup analysis, etiologies-stratified models suggested that the association of

160 hyponatraemia with worse survival was significant among TCL-HLH but not BCL-

161 HLH (Fig. 3A, 3B). Effects were similar across most subgroups (Fig. 3), however, 
there were significant interactions between hyponatraemia and ferritin, TG with respect to poorer overall survival.

\section{Discussion}

To the best of our knowledge, this is the first study to probe into the prognostic effect of serum sodium concentration on the survival of patients with NHL-HLH. we showed that a low level of serum sodium was positively associated with increased mortality, resulting in a linear dose-response relationship.

Hyponatraemia is a common electrolyte disturbance in critical illness and malignancies. Many prospective and retrospective studies have indicated the occurrence of hyponatraemia and its importance as a prognostic factor in ICU patients [16], acute-on-chronic liver failure [17], glioblastoma [18], and lung cancer [19]. Nevertheless, hyponatraemia has rarely been reported during the course of lymphoma, especially NHL-HLH, which has mostly been presented in case reports [20-22]. In this study, we discovered for the first time that the prevalence of hyponatraemia in sHLH patients on admission for NHL was 52.9\%, which was higher than that mentioned above, and moderate to severe hyponatraemia occurred in $20.9 \%$ of patients. Consistent with our results, Zeinah et al discovered that hyponatraemia occurred in $60 \%$ of patients with lymphoma and moderate to severe hyponatraemia occurred in $19 \%$ of patients with lymphoma [23].

Hyponatraemia has been identified as a negative prognostic factor in critical illness and malignancies. A multicentre cohort study between 2005 and 2012 including 7067 participants from 18 ICUs discovered that hyponatraemia was independently associated 
with increased day 28 mortality (OR:1.31, 95\% CI: 1.06 - 1.61) [6]. Jorge and his group showed that compared with normonatraemia, hyponatraemia was associated with significantly worse OS in breast cancer (HR: 3.7, 95\%, CI: 1.9-7.2; $P<0.01$ ), nonsmall-cell lung carcinoma (HR: 2.8, 95\%, CI: 2.0-3.9; $P<0.01$ ) and lymphoma (HR: 4.5, 95\%, CI: $1.8-11.5 ; P<0.01)$ [8]. In keeping with previous results in cancer patients. our present study showed a linear relation between serum sodium and risk of mortality. Hyponatremia on admission were significantly associated with unfavourable OS in NHL-HLH patients (adjusted HR: 1.51, 95\% CI: 1.03-2.20; $P=0.033$ ).

The pathophysiology of hyponatraemia in critical illness as well as haematological malignancies is still not fully understood. Most studies of the occurrence of hyponatraemia and its prognostic value performed to date have been related to central nervous system (CNS) disorders [24], drugs [25], and pulmonary diseases [26], which is usually caused by syndrome of inappropriate antidiuretic hormone secretion (SIADH) and driven by ectopic secretion of arginine vasopressin (AVP) [8, 27]. Nevertheless, in our NHL-HLH cohort, primary CNS lymphoma was not seen in the study; in addition, hyponatraemia occurred prior to treatment. Such high rates of hyponatraemia might also be associated with inflammation. To our knowledge, HLH is characterized by excessive pro-inflammatory cytokines and chemokines, such as IFN- $\gamma$, IL-6, interleukin (IL)-1 and TNF- $\alpha$ [28]. There is a possibility that elevated cytokines could be implicated in the pathogenesis of hyponatraemia in NHL-HLH patients. First, several studies pointed out that high cytokines, such as IL-1 and TNF- $\alpha$, reduce sodium transport by reducing the expression and function of apical epithelial sodium channels 
and/or the sodium-potassium ATPase ( $\mathrm{Na} / \mathrm{K}$ ATPase) at the basolateral membrane, accompanied by increased levels of natriuresis [29, 30]. Second, other studies have revealed that IL-1 $\beta$ and IL-6 injection or elevated levels of IL-6 might increase antidiuretic hormone (ADH) secretion in both animal experiments and human studies, leading to hyponatraemia $[31,32]$. This explanation was verified by SLE patient who improved of hyponatraemia after treatment with tocilizumab (an IL-6 receptor antibody) [33].

Several limitations exist in this study regarding the prognostic impact of serum sodium concentration in NHL-HLH. First, serum sodium concentrations were not corrected for glucose concentrations in this study, while, the impact of this limitation is likely to be small, as diabetes mellitus (DM) patients were documented in only $1.6 \%$ of our patients. Second, cytokine profiles were not available in all patients, and the severity of hyponatraemia and cytokine levels were not verified. Third, we had no information on volume status or measurement of urine electrolytes and urea or diuretic therapy before admission at our hospital. Despite these limitations, the current data are noteworthy because this is the first study, focusing specifically on hyponatraemia at sHLH admission, demonstrating that hyponatraemia is common and is an independent risk factor for a high mortality rate. Therefore, clinicians should recognize hyponatraemia in sHLH.

\section{Conclusion}

We identified that hyponatremia is relatively frequent in patients admitted for NHL-HLH, and it has prognostic implications. Therefore, patients with hyponatremia 
should be carefully monitored.

229

230

231

232

233

234

235

236

237

238

239

240

241

242

243

244

245

246

247

248

249

250

251

\section{Abbreviations}

Non-Hodgkin lymphoma-associated hemophagocytic lymphohistiocytosis, NHL-HLH; sHLH, secondary hemophagocytic lymphohistiocytosis; BC, breast cancer; CRC, colorectal cancer; DM, diabetes mellitus; TG, triglycerides; LDH, lactate dehydrogenase; ALB, albumin; FIB, fibrinogen; sCD25, soluble interleukin-2 receptor;ANC, absolute neutrophil count; HB, hemoglobin; PLT, platelet; ALT, alanine transaminase; AST, aspartate transaminase; $\beta_{2}-\mathrm{MG}$, beta2-microglobulin; EBV, Epstein-Barr virus; MHLH, malignancy-associated hemophagocytic lymphohistiocytosis; Non-MHLH, non-malignancy associated hemophagocytic lymphohistiocytosis; GC, glucocorticoid; IVIG, intravenous immunoglobulins; CsA, cyclosporine; VP16, etoposide; HR, hazards ratio; 95\% CI, 95\% confidence interval; CNS, central nervous system; SIADH, syndrome of inappropriate antidiuretic hormone secretion; AVP, arginine vasopressin; $\mathrm{ADH}$, antidiuretic hormone

\section{Acknowledgements}

The authors express their gratitude for all persons who were involved in reporting on patients and gathering data.

\section{Author contributions}

GLY and HXQ designed the experiments. GLY performed the experiments. GLY, CFM and HXQ organized the clinical materials. GLY and CFM performed the data analysis. GLY and CFM wrote the paper. All authors contributed to the final approval of the manuscript.

\section{Funding}

This study was supported by the National Natural Science Foundation of China (grant 
number 81570175).

\section{Availability of data and materials}

254 The data of our patients is available in the Department of Medical Records at Jiangsu

255 Province Hospital and the First Affiliated Hospital of Nanjing Medical University.

256 These data can be released with consent from the patients and are available from the

257 corresponding author upon reasonable request.

\section{Declarations}

259 Ethics approval and consent to participate

260 Our study was approved by the ethics committee of the First Affiliated Hospital of

261 Nanjing Medical University and registered on the Chinese Clinical Trial Registry 262 (ChiCTR2000032421). All methods were carried out in accordance with relevant 263 guidelines and regulations. Written informed consent was obtained from all patients or 264 legal trustee.

\section{Consent for publication}

266 Not applicable.

\section{Competing interests}

268 The authors declare that they have no competing interests.

269 Author details

$270{ }^{1}$ Department of Geriatric Hematology, The First Affiliated Hospital of Nanjing Medical

271 University, Jiangsu Province Hospital,300 Guangzhou Road, Nanjing 210029, China 


\section{References}

1. Lachmann G, Spies C, Schenk T, Brunkhorst FM, Balzer F, La Rosee P. Hemophagocytic Lymphohistiocytosis: Potentially Underdiagnosed in Intensive Care Units. Shock. 2018;50(2):149-55.

2. Marsh RA, Haddad E. How i treat primary haemophagocytic lymphohistiocytosis. British journal of haematology. 2018;182(2):185-99.

3. Castillo L, Carcillo J. Secondary hemophagocytic lymphohistiocytosis and severe sepsis/ systemic inflammatory response syndrome/multiorgan dysfunction syndrome/macrophage activation syndrome share common intermediate phenotypes on a spectrum of inflammation. Pediatric critical care medicine : a journal of the Society of Critical Care Medicine and the World Federation of Pediatric Intensive and Critical Care Societies. 2009;10(3):387-92.

4. Ramos-Casals M, Brito-Zeron P, Lopez-Guillermo A, Khamashta MA, Bosch X. Adult haemophagocytic syndrome. Lancet. 2014;383(9927):1503-16.

5. Forfia PR, Mathai SC, Fisher MR, Housten-Harris T, Hemnes AR, Champion HC, et al. Hyponatremia predicts right heart failure and poor survival in pulmonary arterial hypertension. American journal of respiratory and critical care medicine. 2008;177(12):1364-9.

6. Darmon M, Pichon M, Schwebel C, Ruckly S, Adrie C, Haouache H, et al. Influence of early dysnatremia correction on survival of critically ill patients. Shock. 2014;41(5):394-9.

7. Il Shin J, Park SJ, Suh CH, Lee GH, Hur MW, Han SY, et al. Hyponatremia in patients with systemic lupus erythematosus. Scientific reports. 2016;6:25566.

8. Castillo JJ, Glezerman IG, Boklage SH, Chiodo J, 3rd, Tidwell BA, Lamerato LE, et al. The occurrence of hyponatremia and its importance as a prognostic factor in a cross-section of cancer patients. BMC cancer. 2016;16:564.

9. Cazzola M. Introduction to a review series: the 2016 revision of the WHO classification of tumors of hematopoietic and lymphoid tissues. Blood. 2016;127(20):2361-4.

10. Liang JH, Wang L, Zhu HY, Qian J, Liao H, Wu JZ, et al. Dose-adjusted EPOCH regimen as first-line treatment for non-Hodgkin lymphoma-associated hemophagocytic lymphohistiocytosis: a single-arm, open-label, phase II trial. Haematologica. 2020;105(1):e29-e32.

11. Henter JI, Horne A, Arico M, Egeler RM, Filipovich AH, Imashuku S, et al. HLH2004: Diagnostic and therapeutic guidelines for hemophagocytic lymphohistiocytosis. Pediatric blood \& cancer. 2007;48(2):124-31.

12. Fardet L, Galicier L, Lambotte O, Marzac C, Aumont C, Chahwan D, et al. Development and validation of the HScore, a score for the diagnosis of reactive hemophagocytic syndrome. Arthritis \& rheumatology. 2014;66(9):2613-20.

13. Andreani G, Carpene E, Cannavacciuolo A, Di Girolamo N, Ferlizza E, Isani G. Reference values for hematology and plasma biochemistry variables, and protein electrophoresis of healthy Hermann's tortoises (Testudo hermanni ssp.). Veterinary clinical pathology. 2014;43(4):573-83. 

hemophagocytic lymphohistiocytosis prognosis is affected by underlying disease and coexisting viral infection: analysis of a single institution series of 35 patients. Hematol Oncol. 2017;35(4):828-34.

15. Yin G, Man C, Liao S, Qiu H. The Prognosis Role of AST/ALT (De Ritis) Ratio in Patients with Adult Secondary Hemophagocytic Lymphohistiocytosis. Mediators Inflamm. 2020;2020:5719751.

16. Pokaharel M, Block CA. Dysnatremia in the ICU. Current opinion in critical care. 2011;17(6):581-93.

17. Cardenas A, Sola E, Rodriguez E, Barreto R, Graupera I, Pavesi M, et al. Hyponatremia influences the outcome of patients with acute-on-chronic liver failure: an analysis of the CANONIC study. Critical care. 2014;18(6):700.

18. Mrowczynski OD, Bourcier AJ, Liao J, Langan ST, Specht CS, Rizk EB. The predictive potential of hyponatremia for glioblastoma patient survival. Journal of neuro-oncology. 2018;138(1):99-104.

19. Fiordoliva I, Meletani T, Baleani MG, Rinaldi S, Savini A, Di Pietro Paolo M, et al. Managing hyponatremia in lung cancer: latest evidence and clinical implications. Therapeutic advances in medical oncology. 2017;9(11):711-9.

20. Ciaudo M, Chauvenet L, Audouin J, Rossert J, Favier R, Horellou MH, et al. Peripheral-T-cell lymphoma with hemophagocytic histiocytosis localised to the bone marrow associated with inappropriate secretion of antidiuretic hormone. Leukemia \& lymphoma. 1995;19(5-6):511-4.

21. Shimizu N, Tanaka S, Watanabe Y, Tokuyama W, Hiruta N, Ohwada C, et al. Syndrome of Inappropriate Antidiuretic Hormone Secretion in a Patient with Mucosaassociated Lymphoid Tissue Lymphoma. Internal medicine. 2017;56(23):3225-9.

22. Watabe R, Shibata K, Hirase N, Kodera T, Muta K, Nishimura J, et al. Angiotropic B-cell lymphoma with hemophagocytic syndrome associated with syndrome of inappropriate secretion of antidiuretic hormone. Annals of hematology. 2000;79(10):581-4.

23. Abu Zeinah GF, Al-Kindi SG, Hassan AA, Allam A. Hyponatraemia in cancer: association with type of cancer and mortality. European journal of cancer care. 2015;24(2):224-31.

24. Lim AKH, Paramaswaran S, Jellie LJ, Junckerstorff RK. A Cross-Sectional Study of Hyponatremia Associated with Acute Central Nervous System Infections. Journal of clinical medicine. 2019;8(11).

25. Liamis G, Filippatos TD, Elisaf MS. Electrolyte disorders associated with the use of anticancer drugs. European journal of pharmacology. 2016;777:78-87.

26. Garcia-Sanz MT, Martinez-Gestoso S, Calvo-Alvarez U, Doval-Oubina L, CambaMatos S, Rabade-Castedo C, et al. Impact of Hyponatremia on COPD Exacerbation Prognosis. Journal of clinical medicine. 2020;9(2).

27. Castillo JJ, Vincent M, Justice E. Diagnosis and management of hyponatremia in cancer patients. The oncologist. 2012;17(6):756-65.

28. Brisse E, Wouters CH, Matthys P. Hemophagocytic lymphohistiocytosis (HLH): A heterogeneous spectrum of cytokine-driven immune disorders. Cytokine \& growth 
359 factor reviews. 2015;26(3):263-80.

360 29. Eisenhut M. Changes in ion transport in inflammatory disease. Journal of 361 inflammation. 2006;3:5.

362 30. Eisenhut M. Changes in renal sodium transport during a systemic inflammatory 363 response. Pediatric nephrology. 2006;21(10):1487-8; author reply 9.

364 31. Palin K, Moreau ML, Sauvant J, Orcel H, Nadjar A, Duvoid-Guillou A, et al. 365 Interleukin-6 activates arginine vasopressin neurons in the supraoptic nucleus during 366 immune challenge in rats. American journal of physiology Endocrinology and 367 metabolism. 2009;296(6):E1289-99.

368 32. Park SJ, Shin JI. Inflammation and hyponatremia: an underrecognized condition? 369 Korean journal of pediatrics. 2013;56(12):519-22.

370 33. Hodax JK, Bialo SR, Yalcindag A. SIADH in Systemic JIA Resolving After 371 Treatment With an IL-6 Inhibitor. Pediatrics. 2018;141(1). 


\section{Figure legends}

Fig. 1 Flowchart of this study.

Fig. 2 A. Crude survival in 153 evaluable patients with NHL-HLH according to the initial value of serum sodium on admission;

Fig. 2 B. Cubic spline plot of the association between serum sodium and the risk of mortality among NHL-HLH. The solid line and dashed line represent the estimated hazard ratios and their corresponding 95\% CIs. Analyses were adjusted for fibrinogen (FIB), ferritin, sCD25 and treatment strategies.

Fig. 3 Overall survival analyses of subgroups of different etiologies, TG, ferritin, FIB, and sCD25 values. TG, triglyceride; FIB, fibrinogen; sCD25, Soluble IL-2 receptor. 
A total of 285 consecutive sHLH patients in our study were admitted to First Affiliated Hospital of Nanjing Medical University between January 2015 and December 2019

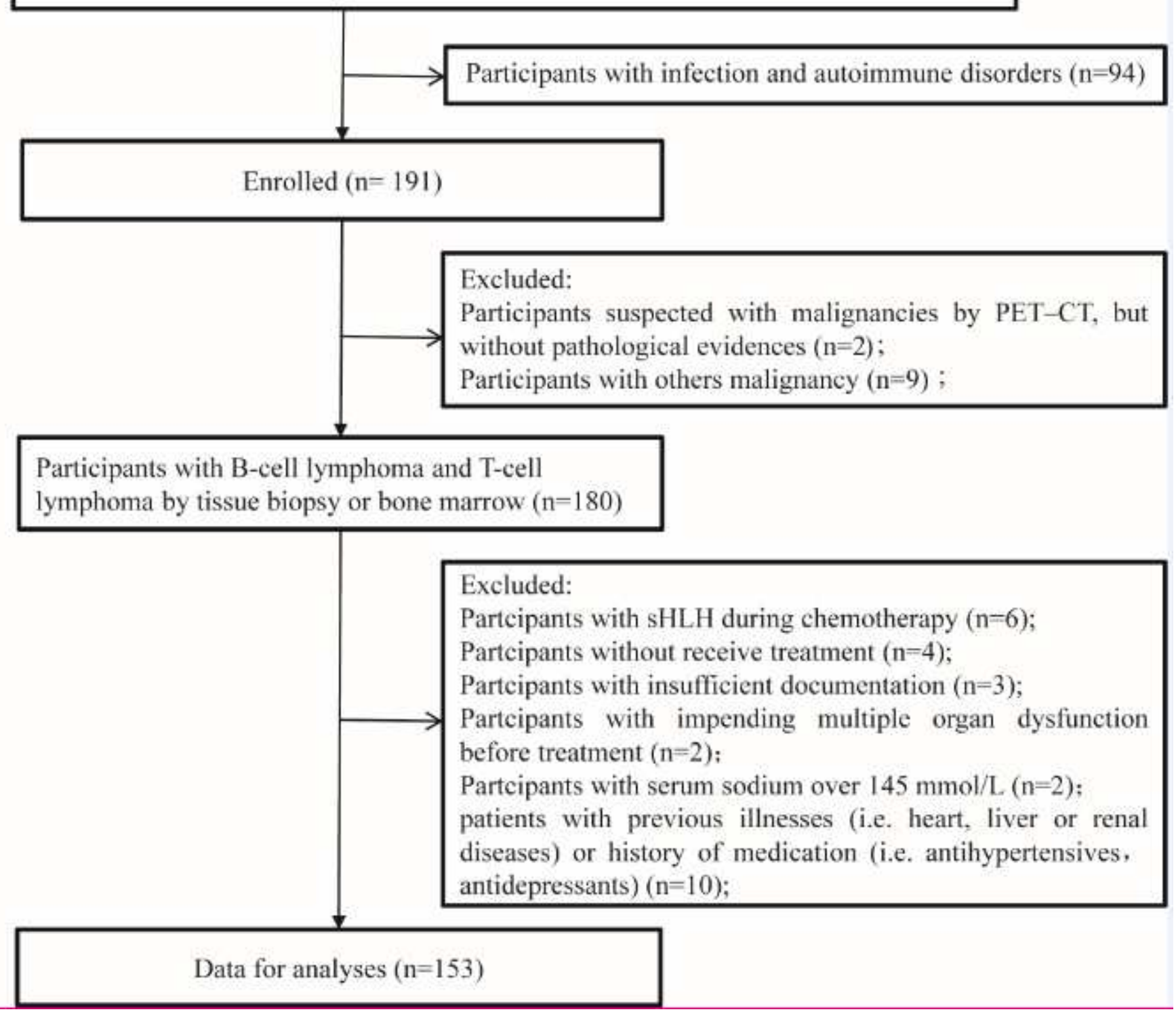

Figure 1

Flowchart of this study. 
A

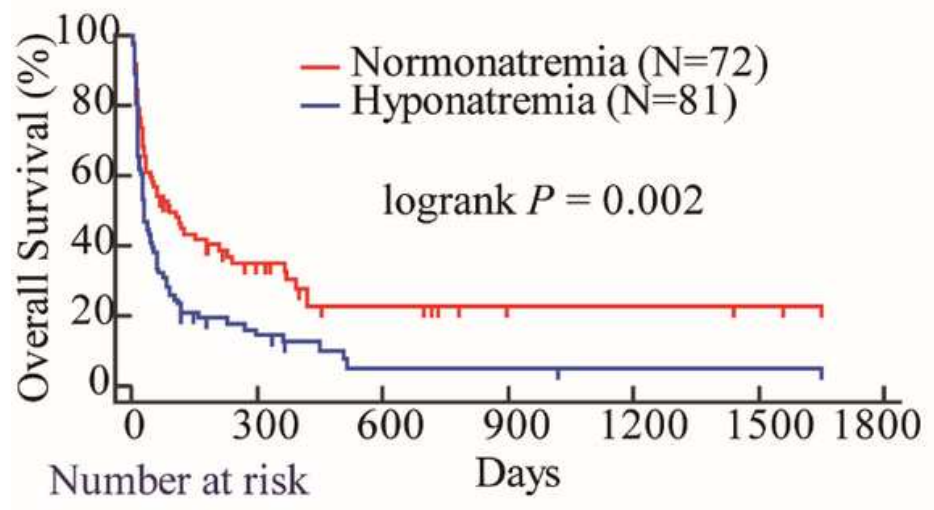

Normonatremia

$\begin{array}{ccccccc}72 & 17 & 8 & 3 & 3 & 2 & 0 \\ \begin{array}{cc}\text { Hyponatremia } \\ 81\end{array} 9 & 2 & 2 & 1 & 1 & 0\end{array}$

B

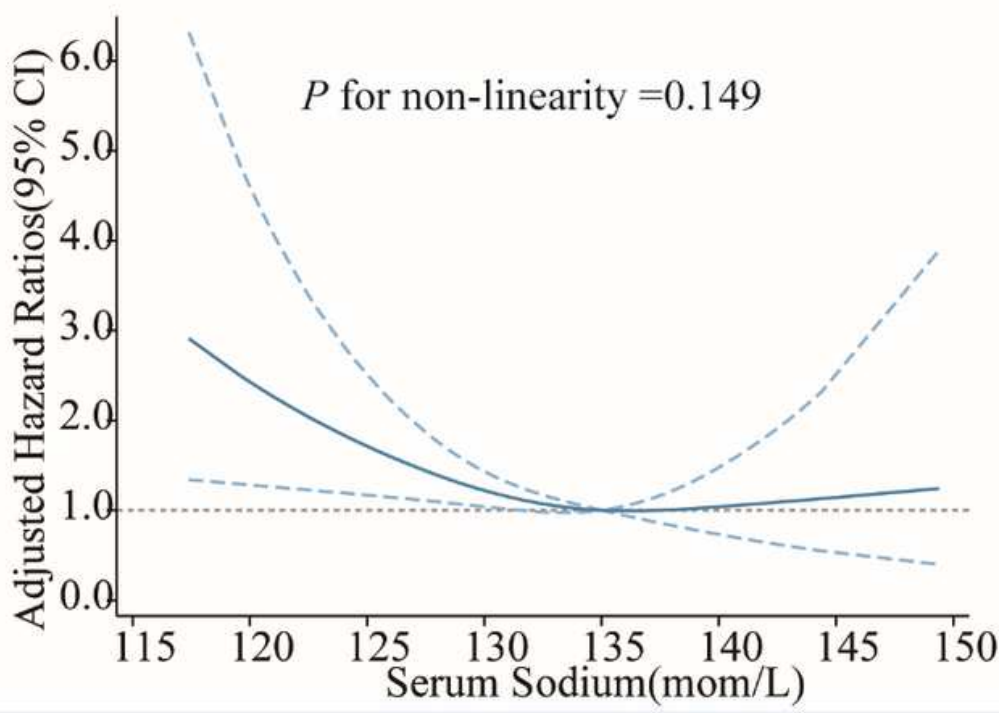

\section{Figure 2}

Fig. 2 A. Crude survival in 153 evaluable patients with NHL-HLH according to the initial value of serum sodium on admission; Fig. 2 B. Cubic spline plot of the association between serum sodium and the risk of mortality among NHL-HLH. The solid line and dashed line represent the estimated hazard ratios and their corresponding 95\% Cls. Analyses were adjusted for fibrinogen (FIB), ferritin, sCD25 and treatment strategies. 
(A) T cell lymphoma

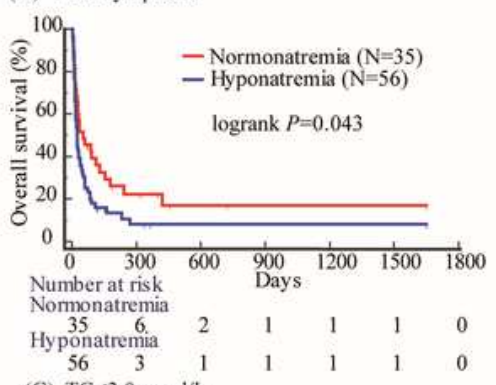

(C) $\mathrm{TG} \leq 3.0 \mathrm{mmol} / \mathrm{L}$

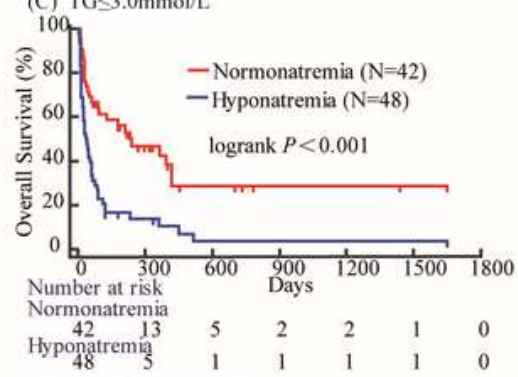

(E) Ferritin $\leq 10000 u g / L$.
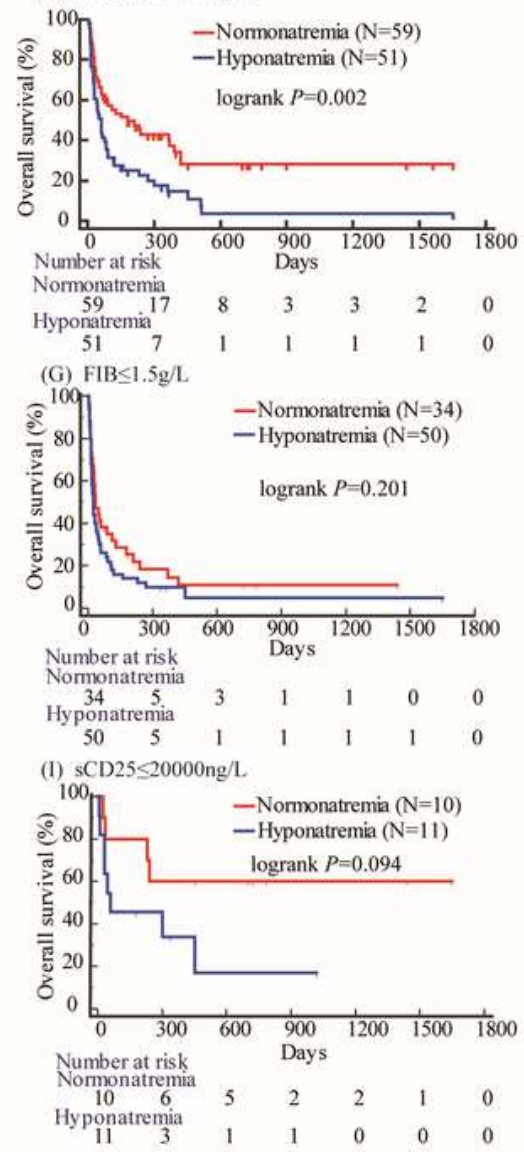

(B) B cell lymphoma
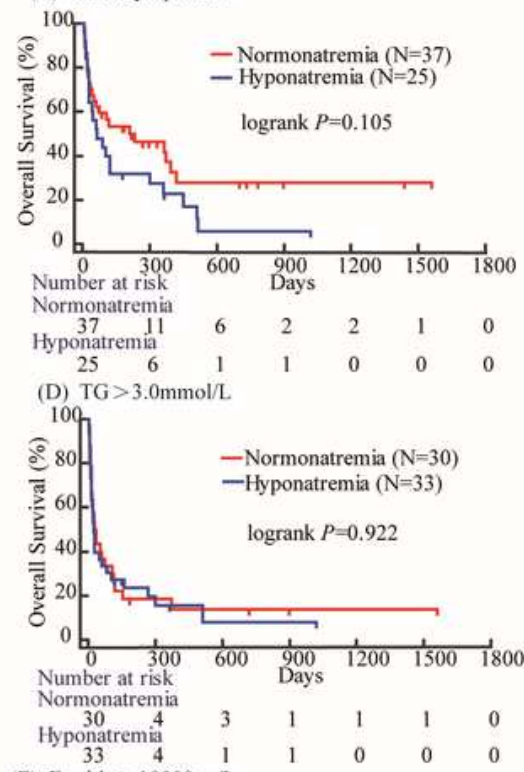

(F) Ferritin $>10000 u g / L$.

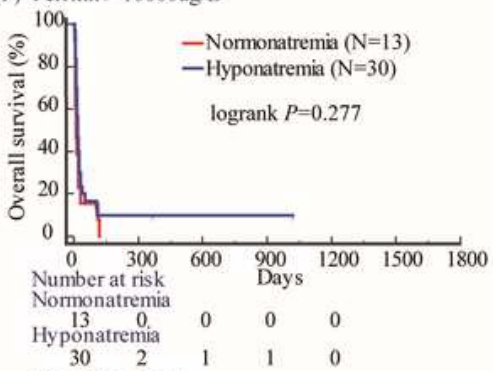

(H) $\mathrm{FIB}>1.5 \mathrm{~g} / \mathrm{L}$

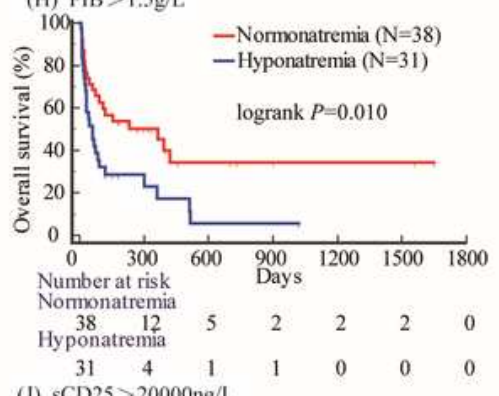

(J) $\mathrm{SCD} 25>20000 \mathrm{ng} / \mathrm{L}$

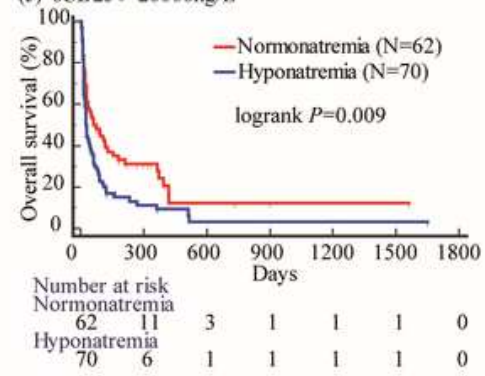

\section{Figure 3}

Overall survival analyses of subgroups of different etiologies, TG, ferritin, FIB, and SCD25 values. TG, triglyceride; FIB, fibrinogen; SCD25, Soluble IL-2 receptor.

\section{Supplementary Files}


This is a list of supplementary files associated with this preprint. Click to download.

- Supplementaryfile1.docx 